\title{
Das Batterieauto ist die Zukunft
}

„Volkswagen spaltet die Autobranche" titelte das Handelsblatt. Im März 2019 hatte VWChef Herbert Diess gedroht, den Lobbyverband der deutschen Autoindustrie (VDA) zu verlassen. Die vom Verband propagierte Fächerstrategie führe in die Sackgasse. Nicht von allem ein bisschen, sondern die Fokussierung auf das Batterieauto sei dringend notwendig. Der Zoff unter den deutschen Autobossen war vorprogrammiert. Volkswagen investiert zweistellige Euro-Milliardenbeträge in das Batterieauto, während BMW und Daimler den schnellen Umstieg nicht schätzen. Macht der VW-Chef einen folgenschweren Fehler oder sind die Münchner und Stuttgarter auf dem Holzweg? Die Fächerstrategie war jahrzehntelang Dogma. Mit dem Dieselmotor, einem Diesotto, dem Brennstoffzellenauto, Power-To-Gas, synthetischen Kraftstoffen, die aus Solarstrom in mehreren chemischen Transformationsprozessen gewonnen werden, mit Biokraftstoffen, etwa aus Raps oder Zuckerrohr hergestellt, mit Plug-In-Hybriden sollte der Weg in die klimaverträgliche Zukunft beschritten werden. Im Mittelpunkt sollte der Verbrennungsmotor stehen, arrondiert mit ein paar Plug-In-Hybriden, Batterie- oder Brennstoffzellenautos: bewährte Technik, kein Abenteuer, abgesicherte Investitionen.

Dann kamen Elon Musk, Dieselgate und die chinesischen Quoten für Elektroautos. Der Chef des US-Autobauers Tesla hat gezeigt, dass ein Batterieauto mit Reichweiten von 500 Kilometern vermarktbar ist. Durch Dieselgate sind die Diesel-Pkw-Verkäufe um fast $50 \%$ eingebrochen. Eine Rückkehr zu den früheren Marktanteilen ist ausgeschlossen. Jetzt schaffen die Autobauer die EU-Klimavorgaben des Jahres 2020 nur, wenn sie $20 \%$ der Neuwagen als Null-Emissionsautos verkaufen. Das sind nach dem heutigen Stand der Technik ausschließlich batterieelektrische Fahrzeuge. Im weltgrößten Automarkt China gilt ab 2019 eine 10\%-Quote für New Energy Vehicles (NEV). NEV sind rein elektrische Autos plus Plug-In-Hybride. Die Quote steigt im Zeitverlauf. Zusätzlich wurden in der EU die Klimavorgaben für das Jahr 2030 verschärft. Ab 2030 darf der $\mathrm{CO}_{2}$-Ausstoß von Neuwagen $60 \mathrm{~g} / \mathrm{km}$ nicht überschreiten. Das entspricht einem Benzinverbrauch von 2,8 l/100 km. Wer mehr verbraucht, muss hohe Strafen zahlen. Nach heutigem Fahrzeugmix müssten dann $50 \%$ der Neuwagen durch NullEmissionsautos ersetzt werden. Das Dilemma der Autobauer wird fühlbar. Null-Emissions-Neuwagen müssen sehr schnell in den Markt. Die Zeit für „Fächerplaudereien“ ist abgelaufen. Das Batterieauto ist alternativlos.

Nicht nur die enge Zeitachse spricht gegen das Brennstoffzellenauto. Der Toyota Mirai, das erste Brennstoffzellenauto in Serie, kostet mehr als 80000 Euro. Aus mitgeführtem Wasserstoff erzeugt die Brennstoffzelle Strom, der das Auto über den Elektromotor antreibt. Der Toyota Mirai hat die Größe eines VW-Golf. Niemand würde einen VW-Golf für 80000 Euro kaufen. Selbst mit größten Anstrengungen braucht es mehr als 30 Jahre, bis der Preis das Niveau von Batterieautos erreicht. Hinzu kommt, dass Brennstoffzellen "gleichmäßigen“ Strom erzeugen. Das Brennstoffzellenauto braucht daher für Spitzenleistungen - etwa bei Überholvorgängen - zusätzliche Batterien. Bleibt die Tankinfrastruktur. Wasserstoff wird heute überwiegend aus fossilen Energieträgern gewonnen. Der Umstieg auf regenerative Erzeugung ist zeit- und kostenintensiv. Die Kosten einer zweiten Infrastruktur, neben der Elektro-Ladeinfrastruktur, liegen im Euro-Milliardenbereich. Zusätzlich müsste in den ersten zehn Jahren mit hohen Kosten für nicht genutzte Tankinfrastruktur-Kapazitäten gerechnet werden. Niemand kauft sich ein Brennstoffzellenauto, wenn es kein Tankstellennetz gibt. Also muss zuerst selbst für wenige Autos die Tankinfrastruktur stehen. Schnell wäre der Ruf nach dem Staat wieder da. In einer netten Anekdote hat Daimler-Chef Dieter Zet-

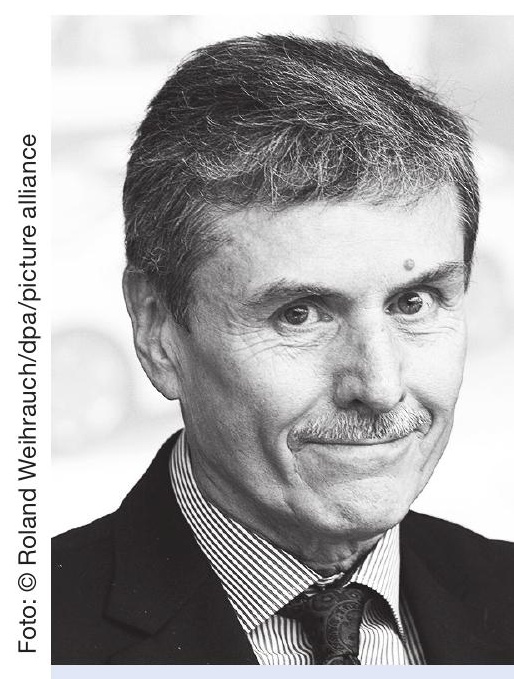

Ferdinand Dudenhöffer ist Direktor des CARInstituts an der Universität Duisburg-Essen sowie dort Inhaber des Lehrstuhls für allgemeine $\mathrm{Be}$ triebswirtschaftslehre und Automobilwirtschaft. 
sche die Frage nach dem Brennstoffzellenauto auf den Punkt gebracht. Zetsche saß mit Tesla-Chef Elon Musk beim Frühstück. Zetsche fragte den Technik-Freak Musk, wie er das Brennstoffzellenauto einschätzt. „Dieter, ich kann Dir nicht sagen, welches die bessere Technik ist. Aber ich weiß, dass die Ladeinfrastruktur für Elektroautos steht und die Produktion läuft.“, stellte Elon Musk fest. Das Rennen ist gelaufen.

Dieselgate war das Symptom, die Ursache liegt tiefer. Multiples Politikversagen hat Deutschlands Vorzeigeindustrie in eine prekäre Lage gebracht. Mehr als 20 Jahre lang wurden aufgrund von EU-Verordnungen die Angaben zum Kraftstoffverbrauch und $\mathrm{zu} \mathrm{CO} \mathrm{CO}_{2}$-Emissionen von Neuwagen falsch ausgewiesen. Mit der Messvorgabe „Neuer Europäischer Fahrzyklus“ (NEFZ) hatte die EU Vorschriften definiert, die Kraftstoffverbrauch und $\mathrm{CO}_{2}$-Emissionen von Neuwagen bei „normaler" Fahrt um mehr als $20 \%$ zu niedrig angeben. Das war allen bewusst. Zwar ist mittlerweile die neue Messvorschrift WLTP eingeführt, aber die $\mathrm{CO}_{2}$-Grenzwerte werden auf den NEFZ umgerechnet. Das wird nicht ewig funktionieren. Zusätzlich ist der tatsächliche $\mathrm{CO}_{2}$-Ausstoß im Straßenverkehr, verursacht durch parlamentarisch verabschiedete falsche Messvorschriften, deutlich höher als prognostiziert. Die willkürliche DieselBevorzugung bei der Kraftstoffsteuer ist seit langen Jahren nicht nur dem Bundesrechnungshof berechtigterweise ein Dorn im Auge. Die Bundesfinanzminister weigern sich renitent seit 50 Jahren, die staatliche Preisverzerrung zu beseitigen. Wenn Dieselkraftstoff aus fossilen Energieträgern steuerlich subventioniert wird, legt man dem Null-Emissions-Auto Steine in den Weg. Statt klare Lösungen vorzugeben, werden zusätzliche Kaufprämien für Elektroautos ausgelobt. „Das eine tun, ohne das andere zu lassen“, nennt das die Bundeskanzlerin. Als ob man gleichzeitig mit einem Batterieauto und einem Diesel-Pkw fahren könnte. Es klingt schizophren.

Seit einiger Zeit bezahlt die Bundesregierung Fördergelder für das Aufstellen von Ladesäulen. Einen Art Masterplan für Europa - wo Schnellladesäulen notwendig sind, wo normale Ladesäulen ausreichend sind, in welchem Tempo ausgebaut werden muss, damit eine Art Balance zwischen Zahl der Elektroautos und der Ladepunkte existiert - gibt es nicht. Stattdessen wird gegen Antragsformular beliebig Steuergeld ausbezahlt. Dass dieses chaotische System zu großen Fehlern neigt und teure Infrastruktur an falschen Standorten aufbaut, liegt auf der Hand. Politikversagen erzeugt Investitionsruinen.

Bundeswirtschaftsminister Peter Altmaier hat hochfliegende Pläne. Fabriken für Batteriezellen müssten her, sonst verliere Europas Autoindustrie den Anschluss, mahnen Berlin und Brüssel. Mehr als zehn globale Unternehmen, wie CATL, LG-Chem, Panasonic, Samsung, SK-Innovation, haben sich in Südkorea, China und Japan auf die Zellproduktion spezialisiert. Die Produktion macht weniger als $20 \%$ der Wertschöpfung der Zelle aus und ist hochautomatisiert. Arbeitsplätze gibt es für Roboter. Wo da in einer globalen Welt, die Ricardos komparative Vorteile vor Augen hat, strategische Abhängigkeiten sein sollen, verstehe wer will. Statt in Forschung für nächste BatterieGenerationen, in Elektrochemie an deutschen Hochschulen und Forschungsinstituten zu investieren, werden mit Steuergeld Steine für den Nachbau von Fabriken aufeinandergelegt.

Die Serie falscher Allokationsentscheidungen lässt sich fortsetzen, etwa mit der EEG-Umlage, die Deutschland international die höchsten Strompreise gebracht hat. Der Ausbau der Stromnetze von Nord- nach Süddeutschland bleibt eine Baustelle. Politischer Zick-Zack-Kurs und Wahltaktik sind die Ursachen für das Hinterherhinken

Ferdinand Dudenhöffer

Universität Duisburg-Essen

ferdinand.dudenhoeffer@uni-due.de der Branche. Der Systemfehler liegt im Politikbereich. Das Dilemma nach Fukushima, der Ausstieg aus der Atomenergie in einer Nacht-und-Nebel-Aktion, wiederholt sich in der Verkehrswende. Nur sind die Kosten um ein Vielfaches höher. 